\title{
Nontrivial Solutions for Asymmetric Kirchhoff Type Problems
}

\author{
Ruichang Pei ${ }^{1}$ and Jihui Zhang ${ }^{2}$ \\ ${ }^{1}$ School of Mathematics and Statistics, Tianshui Normal University, Tianshui 741001, China \\ ${ }^{2}$ Institute of Mathematics, School of Mathematics and Computer Sciences, Nanjing Normal University, Nanjing 210097, China
}

Correspondence should be addressed to Ruichang Pei; prc211@163.com

Received 26 December 2013; Accepted 24 March 2014; Published 16 April 2014

Academic Editor: Mihai Mihǎilescu

Copyright (C) 2014 R. Pei and J. Zhang. This is an open access article distributed under the Creative Commons Attribution License, which permits unrestricted use, distribution, and reproduction in any medium, provided the original work is properly cited.

We consider a class of particular Kirchhoff type problems with a right-hand side nonlinearity which exhibits an asymmetric growth at $+\infty$ and $-\infty$ in $\mathbb{R}^{N}(N=2,3)$. Namely, it is 4 -linear at $-\infty$ and 4 -superlinear at $+\infty$. However, it need not satisfy the Ambrosetti-Rabinowitz condition on the positive semiaxis. Some existence results for nontrivial solution are established by combining Mountain Pass Theorem and a variant version of Mountain Pass Theorem with Moser-Trudinger inequality.

\section{Introduction}

We consider the following nonlocal Kirchhoff type problem:

$$
\begin{gathered}
-\left(1+\int_{\Omega}|\nabla u|^{2}\right) \Delta u(x)=f(x, u), \quad \text { in } \Omega, \\
u=0, \quad \text { on } \partial \Omega,
\end{gathered}
$$

where $\Omega$ is a smooth bounded domain in $R^{N}(N=2,3)$ and $f: \bar{\Omega} \times R \rightarrow R$ is continuous.

It is pointed out in [1] that the problem (1) models several physical and biological systems where $u$ describes a process which depends on the average of itself (e.g., population density). Moreover, this problem is related to the stationary analogue of the Kirchhoff equation

$$
u_{t t}-\left(1+\int_{\Omega}|\nabla u|^{2}\right) \Delta u=g(x, t),
$$

which was proposed by Kirchhoff [2] as an extension of the classical D'Alembert wave equation for free vibration of elastic strings. Kirchhoff's model takes into account the changes in length of the string produced by transverse vibrations. Some early studies of the Kirchhoff equation may be seen [3-5]. More recently, by variational methods, Alves et al. [1] and Ma and Rivera [6] studied the existence of one positive solution, and $\mathrm{He}$ and Zou [7] studied the existence of infinitely many positive solutions for the problem (1), respectively; Perera and Zhang [8] studied the existence of nontrivial solutions for the problem (1) via the Yang index theory; Zhang and Perera [9] and Mao and Zhang [10] studied the existence of sign-changing solutions for the problem (1) via invariant sets of descent flow. In particular, the asymptotically 4 -linear case,

$$
\lim _{t \rightarrow 0} \frac{f(x, t)}{t}=\lambda, \quad \lim _{t \rightarrow+\infty} \frac{f(x, t)}{t^{3}}=\mu \text { uniformly in } x,
$$

was considered in [8]. In [9], the authors considered the 4superlinear case:

$$
\text { (AR) } \exists v>4: v F(x, t) \leq t f(x, t), \quad|t| \text { large, }
$$

where $F(x, t)=\int_{0}^{t} f(x, s) d s$, which implies that there exists a constant $c>0$ such that

$$
F(x, t) \geq c\left(|t|^{\nu}-1\right) .
$$

Note that (AR) condition plays an important role for showing the boundedness of Palais-Smale sequences. Furthermore, by a simple calculation, it is easy to see that (AR) condition implies that

$$
\lim _{t \rightarrow+\infty} \frac{F(x, t)}{t^{4}}=+\infty .
$$

Hence $F(x, u)$ grows in a 4 -superlinear rate as $|u| \rightarrow+\infty$.

In the present paper, motivated by [11-14], our main purpose is to establish existence results of nontrivial solution 
for the problem (1) with $N=2,3$ when the nonlinearity term $f(x, \cdot)$ exhibits an asymmetric behavior as $t \in \mathbb{R}$ approaches $+\infty$ and $-\infty$. More precisely, we assume that, for a.e. $x \in$ $\Omega, f(x, \cdot)$ grows 4 -superlinear at $+\infty$, while at $-\infty$ it has a 4 linear growth. To our knowledge, this asymmetric nonlocal Kirchhoff problem is rarely considered by other people.

In case of $N=3$, all the above-mentioned works involve the nonlinear term $f(x, u)$ of a subcritical (polynomial) growth; say,

(SCP): there exist positive constants $c_{1}$ and $c_{2}$ and $q_{0} \in$ $(3,5)$ such that

$$
|f(x, t)| \leq c_{1}+c_{2}|t|^{q_{0}}, \quad \forall t \in \mathbb{R}, x \in \Omega .
$$

One of the main reasons to assume this condition (SCP) is that they can use the Sobolev compact embedding $H_{0}^{1}(\Omega) \hookrightarrow$ $L^{q}(\Omega), 1 \leq q<6$.

Over the years, many researchers studied the problem (1) by trying to drop the condition (AR); see, for instance, $[8,15]$.

In this paper, our first main results will be to study the problem (1) in the improved subcritical polynomial growth as follows:

$$
\text { (SCPI) : } \lim _{t \rightarrow \infty} \frac{f(x, t)}{t^{5}}=0
$$

which is much weaker than (SCP). Note that, in this case, we do not have the Sobolev compact embedding anymore. Our work is studying the asymmetric problem (1) without the (AR) condition in the positive semiaxis. In fact, this condition was studied by Liu and Wang in [16] in the case of Laplacian (i.e., $p=2$ ) by the Nehari manifold approach. However, we will use the Mountain Pass Theorem and a suitable version of the Mountain Pass Theorem to get the nontrivial solution to the problem (1) in the case that $N=3$. Our results are different from those in $[8-10,15]$.

Let us now state our results. Suppose that $f(x, t) \in C(\bar{\Omega} \times$ $\mathbb{R})$ and satisfies

$\left(H_{1}\right) \lim _{t \rightarrow 0}(f(x, t) / t)=f_{0}$ uniformly, for a.e. $x \in \Omega$, where $f_{0} \in[0,+\infty)$;

$\left(H_{2}\right) \lim _{t \rightarrow-\infty}\left(f(x, t) / t^{3}\right)=l$ uniformly, for a.e. $x \in \Omega$, where $l \in[0,+\infty]$;

$\left(H_{3}\right) \lim _{t \rightarrow+\infty}\left(f(x, t) / t^{3}\right)=+\infty$ uniformly, for a.e. $x \in \Omega$;

$\left(H_{4}\right)\left(f(x, t) / t^{3}\right)$ is nonincreasing with respect to $t \leq 0$, for a.e. $x \in \Omega$.

We need the following preliminaries.

Let $E:=H_{0}^{1}(\Omega)$ be the Sobolev space equipped with the inner product and the norm

$$
\langle u, v\rangle=\int_{\Omega} \nabla u \nabla v d x, \quad\|u\|=\langle u, u\rangle^{1 / 2} .
$$

We denote by $|\cdot|_{p}$ the usual $L^{p}$-norm. Since $\Omega$ is a bounded domain, $E \hookrightarrow L^{p}(\Omega)$ continuously for $p \in[1,6]$, compactly for $p \in[1,6)$, and there exists $\gamma_{p}>0$ such that

$$
|u|_{p} \leq \gamma_{p}\|u\|, \quad \forall u \in E .
$$

Recall that function $u \in E$ is called a weak solution of (1) if

$$
\left(1+\|u\|^{2}\right) \int_{\Omega} \nabla u \nabla v d x=\int_{\Omega} f(x, u) v d x, \quad \forall v \in E .
$$

Seeking a weak solution of the problem (1) is equivalent to finding a critical point $u^{*}$ of $C^{1}$ functional as follows:

$$
I(u):=\frac{1}{2}\|u\|^{2}+\frac{1}{4}\|u\|^{4}-\int_{\Omega} F(x, u) d x, \quad \forall u \in E,
$$

where $F(x, u)=\int_{0}^{u} f(x, s) d s$. Then

$$
\begin{aligned}
\left\langle I^{\prime}\left(u^{*}\right), v\right\rangle & =\left(1+\left\|u^{*}\right\|^{2}\right) \int_{\Omega} \nabla u^{*} \nabla v-\int_{\Omega} f\left(x, u^{*}\right) v d x \\
& =0, \quad \forall v \in E .
\end{aligned}
$$

Definition 1. Let $\left(E,\|\cdot\|_{E}\right)$ be a real Banach space with its dual space $\left(E^{*},\|\cdot\|_{E^{*}}\right)$ and $I \in C^{1}(E, \mathbb{R})$. For $c \in \mathbb{R}$, one says that $I$ satisfies the $(\mathrm{PS})_{c}$ condition if, for any sequence $\left\{x_{n}\right\} \subset E$ with

$$
I\left(x_{n}\right) \longrightarrow c, \quad D I\left(x_{n}\right) \longrightarrow 0 \quad \text { in } E^{*},
$$

there is a subsequence $\left\{x_{n_{k}}\right\}$ such that $\left\{x_{n_{k}}\right\}$ converges strongly in $E$. Also, one says that $I$ satisfy the $(C)_{c}$ condition if, for any sequence $\left\{x_{n}\right\} \subset E$ with

$$
I\left(x_{n}\right) \longrightarrow c, \quad\left\|D I\left(x_{n}\right)\right\|_{E^{*}}\left(1+\left\|x_{n}\right\|_{E}\right) \longrightarrow 0,
$$

there is subsequence $\left\{x_{n_{k}}\right\}$ such that $\left\{x_{n_{k}}\right\}$ converges strongly in $E$.

We have the following version of the Mountain Pass Theorem (see $[17,18])$.

Proposition 2. Let $E$ be a real Banach space and suppose that $I \in C^{1}(E, R)$ satisfies the condition

$$
\max \left\{I(0), I\left(u_{1}\right)\right\} \leq \alpha<\beta \leq \inf _{\|u\|=\rho} I(u),
$$

for some $\alpha<\beta, \rho>0$, and $u_{1} \in E$ with $\left\|u_{1}\right\|>\rho$. Let $c \geq \beta$ be characterized by

$$
c=\inf _{\gamma \in \Gamma} \max _{0 \leq t \leq 1} I(\gamma(t)),
$$

where $\Gamma=\left\{\gamma \in C([0,1], E), \gamma(0)=0, \gamma(1)=u_{1}\right\}$ is the set of continuous paths joining 0 and $u_{1}$. Then, there exists a sequence $\left\{u_{n}\right\} \subset$ E such that

$$
\begin{aligned}
I\left(u_{n}\right) \longrightarrow c \geq \beta, \quad\left(1+\left\|u_{n}\right\|\right)\left\|I^{\prime}\left(u_{n}\right)\right\|_{E^{*}} & \longrightarrow 0 \\
\text { as } n & \longrightarrow \infty .
\end{aligned}
$$

Lastly, we also need the following preparations.

Our assumptions lead us to the eigenvalue problem

$$
\begin{gathered}
-\|u\|^{2} \Delta u=\mu u^{3}, \quad \text { in } \Omega, \\
u=0, \quad \text { on } \partial \Omega,
\end{gathered}
$$


where $\mu$ is an eigenvalue of the problem (19) meaning that there is a nonzero $u \in E$ such that

$$
\|u\|^{2} \int_{\Omega} \nabla u \nabla v d x=\mu \int_{\Omega} u^{3} v d x, \quad \forall v \in E .
$$

This $u$ is called an eigenvector corresponding to eigenvalue $\mu$. Set

$$
I(u)=\|u\|^{4}, \quad u \in S:=\left\{u \in E: \int_{\Omega} u^{4}=1\right\} .
$$

Denote by $0<\mu_{1}<\mu_{2}<\cdots$ all distinct eigenvalues of the nonlinear problem (19). Then

$$
\mu_{1}:=\inf _{u \in S} I(u)
$$

where $\mu_{1}>0$ is simple and isolated and $\mu_{1}$ can be achieved at some $\psi_{1} \in S$ and $\psi_{1}>0$ in $\Omega$ (see [9]).

Theorem 3. Let $N=3$ and assume that $f$ has the improved subcritical polynomial growth on $\Omega$ (condition (SCPI)) and satisfies $\left(H_{1}\right)-\left(H_{3}\right)$. If $f_{0}<\lambda_{1}\left(\lambda_{1}>0\right.$ is the first eigenvalue of $(-\Delta, E))$ and $\mu_{1}<l<\infty$, then the problem (1) has at least one nontrivial solution when $l \neq \mu_{i}$, for all $i \in \mathbb{N}$.

Theorem 4. Let $N=3$ and assume that $f$ has the improved subcritical polynomial growth on $\Omega$ (condition (SCPI)) and satisfies $\left(H_{1}\right)-\left(H_{3}\right)$. If $f_{0}<\lambda_{1}\left(\lambda_{1}>0\right.$ is the first eigenvalue of $(-\Delta, E)), l=\mu_{1}$, and $\lim _{t \rightarrow-\infty}[f(x, t) t-4 F(x, t)]=+\infty$ uniformly, for a.e. $x \in \Omega$, then the problem (1) has at least one nontrivial solution.

Here, we also give an example for $f(x, t)$. It satisfies our conditions $\left(H_{1}\right)-\left(H_{3}\right)$ and (SCPI).

Example A. Define

$$
f(x, t)= \begin{cases}g(t)|t|^{2} t+Q(t), & t \leq 0 \\ g(t)|t|^{2} t+h(t), & t>0\end{cases}
$$

where $g(t) \in C(R), g(0)=0 ; g(t) \geq 0, t \in \mathbb{R}$; $h(t) \in C[0,+\infty) ; \lim _{t \rightarrow+0}\left(h(t) / t^{3}\right)=0 ; \lim _{t \rightarrow+\infty}\left(h(t) / t^{5}\right)=$ $0 ; \lim _{t \rightarrow+\infty}\left(h(t) / t^{3}\right)=+\infty ; Q(t) \quad \in C(-\infty, 0]$; $\lim _{t \rightarrow-0}\left(Q(t) / t^{3}\right)=0 ; \lim _{t \rightarrow-\infty}\left(Q(t) / t^{2}\right)=-1$. Moreover, there exists $t_{0}>0$ such that $g(t) \equiv \mu_{1}$ for all $|t| \geq t_{0}$.

Theorem 5. Let $N=3$ and assume that $f$ has the improved subcritical polynomial growth on $\Omega$ (condition (SCPI)) and satisfies $\left(H_{1}\right)-\left(H_{4}\right)$. If $f_{0}<\lambda_{1}\left(\lambda_{1}>0\right.$ is the first eigenvalue of $(-\Delta, E)$ ) and $l=+\infty$, then the problem (1) has at least one nontrivial solution.

In case of $N=2$, we have $2^{*}=+\infty$. In this case, every polynomial growth is admitted, but one knows easy examples that $E \nsubseteq L^{\infty}(\Omega)$. Hence, one is led to look for a function $g(s)$ : $\mathbb{R} \rightarrow R^{+}$with maximal growth such that

$$
\sup _{u \in E,\|u\| \leq 1} \int_{\Omega} g(u) d x<\infty
$$

It was shown by Trudinger [19] and Moser [20] that the maximal growth is of exponential type. So we must redefine the subcritical (exponential) growth in this case as follows.

(SCE): $f$ has subcritical (exponential) growth on $\Omega$; that is, $\lim _{t \rightarrow \infty}\left(|f(x, t)| / \exp \left(\alpha|t|^{2}\right)\right)=0$ uniformly on $x \in \Omega$ for all $\alpha>0$.

When $N=2$ and $f$ has the subcritical (exponential) growth (SCE), our work is again studying the asymmetric problem (1) without the (AR) condition in the positive semiaxis. Our results are as follows.

Theorem 6. Let $N=2$ and assume that $f$ has the subcritical exponential growth on $\Omega$ (condition (SCE)) and satisfies $\left(H_{1}\right)-\left(H_{3}\right)$. If $f_{0}<\lambda_{1}\left(\lambda_{1}>0\right.$ is the first eigenvalue of $\left.(-\Delta, E)\right)$ and $\mu_{1}<l<\infty$, then the problem (1) has at least one nontrivial solution when $l \neq \mu_{i}$, for all $i \in \mathbb{N}$.

Theorem 7. Let $N=2$ and assume that $f$ has the subcritical exponential growth on $\Omega$ (condition (SCE)) and satisfies $\left(H_{1}\right)-\left(H_{3}\right)$. If $f_{0}<\lambda_{1}\left(\lambda_{1}>0\right.$ is the first eigenvalue of $(-\Delta, E)), l=\mu_{1}$, and $\lim _{t \rightarrow-\infty}[f(x, t) t-4 F(x, t)]=+\infty$ uniformly, for a.e. $x \in \Omega$, then the problem (1) has at least one nontrivial solution.

Theorem 8. Let $N=2$ and assume that $f$ has the subcritical exponential growth on $\Omega$ (condition (SCE)) and satisfies $\left(H_{1}\right)-\left(H_{4}\right)$. If $f_{0}<\lambda_{1}\left(\lambda_{1}>0\right.$ is the first eigenvalue of $(-\Delta, E)$ ) and $l=+\infty$, then the problem (1) has at least one nontrivial solution.

\section{Some Lemmas}

Lemma 9. Let $N=3$ and let $\psi_{1}>0$ be a $\mu_{1}$ eigenfunction with $\left\|\psi_{1}\right\|=1$ and assume that $\left(H_{1}\right)-\left(H_{3}\right)$ and (SCPI) hold. If $f_{0}<\lambda_{1}\left(\lambda_{1}>0\right.$ is the first eigenvalue of $\left.(-\Delta, E)\right)$ and $\mu_{1}<l<$ $\infty$, then

(i) there exist $\rho, \alpha>0$ such that $I(u) \geq \alpha$, for all $u \in E$ with $\|u\|=\rho$;

(ii) $I\left(t \psi_{1}\right) \rightarrow-\infty$ as $t \rightarrow+\infty$.

Proof. By (SCPI) and $\left(H_{1}\right)-\left(H_{3}\right)$, if $l \in\left(\mu_{1},+\infty\right)$, for any $\varepsilon>$ 0 , there exist $A_{1}=A_{1}(\varepsilon)$ and $B_{1}=B_{1}(\varepsilon)$ such that, for all $(x, s) \in \Omega \times \mathbb{R}$,

$$
\begin{gathered}
F(x, s) \leq \frac{1}{2}\left(f_{0}+\varepsilon\right)|s|^{2}+A_{1}|s|^{6}, \\
F(x, s) \geq \frac{1}{4}(l-\varepsilon)|s|^{4}-B_{1} \quad \text { if } l \in\left(\mu_{1},+\infty\right) .
\end{gathered}
$$

Choose $\varepsilon>0$ such that $\left(f_{0}+\varepsilon\right)<\lambda_{1}$. By (25), the Poincaré inequality, and the Sobolev inequality, $|u|_{6}^{6} \leq K\|u\|^{6}$, we get

$$
\begin{aligned}
I(u) & \geq \frac{1}{2}\|u\|^{2}+\frac{1}{4}\|u\|^{4}-\frac{f_{0}+\varepsilon}{2}|u|_{2}^{2}-A_{1}|u|_{6}^{6} \\
& \geq \frac{1}{2}\left(1-\frac{f_{0}+\varepsilon}{\lambda_{1}}\right)\|u\|^{2}-A_{1} K\|u\|^{6} .
\end{aligned}
$$

So part (i) is proved if we choose $\|u\|=\rho>0$ small enough. 
On the other hand, if $l \in\left(\mu_{1},+\infty\right)$, taking $\varepsilon>0$ such that $l-\varepsilon>\mu_{1}$ and using (26), we have

$$
\begin{aligned}
I\left(t \psi_{1}\right) \leq & \frac{1}{2} t^{2}\left\|\psi_{1}\right\|^{2}+\frac{1}{4}\left(1-\frac{l-\varepsilon}{\mu_{1}}\right) t^{4}\left\|\psi_{1}\right\|^{4} \\
& +B_{1}|\Omega| \longrightarrow-\infty \text { as } t \longrightarrow-\infty .
\end{aligned}
$$

Thus part (ii) is proved. By exactly slight modification to the proof above, we can prove (ii) if $l=+\infty$.

Lemma 10 (see $[19,20])$. Let $u \in W_{0}^{1,2}(\Omega)$; then $\exp \left(|u|^{2}\right) \in$ $L^{q}(\Omega)$, for all $1 \leq q<\infty$. Moreover,

$$
\lim _{u \in E,\|u\| \leq 1} \int_{\Omega} \exp \left(\alpha|u|^{2}\right) d x \leq C(\Omega) \quad \text { for } \alpha \leq \alpha_{2}=4 \pi^{2} .
$$

The inequality is optimal; for any growth $\exp \left(\alpha|u|^{2}\right)$ with $\alpha>$ $\alpha_{2}$ the corresponding supremum is $+\infty$.

Lemma 11. Let $N=2$ and let $\psi_{1}>0$ be a $\mu_{1}$ eigenfunction with $\left\|\psi_{1}\right\|=1$ and assume that $\left(H_{1}\right)-\left(H_{3}\right)$ and (SCE) hold. If $f_{0}<\lambda_{1}\left(\lambda_{1}>0\right.$ is the first eigenvalue of $\left.(-\Delta, E)\right)$ and $\mu_{1}<$ $l<\infty$, then

(i) there exist $\rho, \alpha>0$ such that $I(u) \geq \alpha$, for all $u \in E$ with $\|u\|=\rho$;

(ii) $I\left(t \psi_{1}\right) \rightarrow-\infty$ as $t \rightarrow+\infty$.

Proof. By (SCE) and $\left(H_{1}\right)-\left(H_{3}\right)$, if $l \in\left(\mu_{1},+\infty\right)$, for any $\varepsilon>$ 0 , there exist $A_{1}=A_{1}(\varepsilon), B_{1}=B_{1}(\varepsilon), \kappa>0$, and $q>4$ such that, for all $(x, s) \in \Omega \times \mathbb{R}$,

$$
\begin{gathered}
F(x, s) \leq \frac{1}{2}\left(f_{0}+\varepsilon\right)|s|^{2}+A_{1} \exp \left(\kappa|s|^{2}\right)|s|^{q}, \\
F(x, s) \geq \frac{1}{4}(l-\varepsilon)|s|^{4}-B_{1} \quad \text { if } l \in\left(\mu_{1},+\infty\right) .
\end{gathered}
$$

Choose $\varepsilon>0$ such that $\left(f_{0}+\varepsilon\right)<\lambda_{1}$. By (30), the Holder inequality, and the Moser-Trudinger embedding inequality, we get

$$
\begin{aligned}
I(u) \geq & \frac{1}{2}\|u\|^{2}-\frac{f_{0}+\varepsilon}{2}|u|_{2}^{2}+\frac{1}{4}\|u\|^{4} \\
& -A_{1} \int_{\Omega} \exp \left(\kappa|u|^{2}\right)|u|^{q} d x \\
\geq & \frac{1}{2}\left(1-\frac{f_{0}+\varepsilon}{\lambda_{1}}\right)\|u\|^{2}+\frac{1}{4}\|u\|^{4} \\
& -A_{1}\left(\int_{\Omega} \exp \left(\kappa r\|u\|^{2}\left(\frac{|u|}{\|u\|}\right)^{2}\right) d x\right)^{1 / r} \\
& \times\left(\int_{\Omega}|u|^{r^{\prime} q} d x\right)^{1 / r^{\prime}} \\
\geq & \frac{1}{2}\left(1-\frac{f_{0}+\varepsilon}{\lambda_{1}}\right)\|u\|^{2}+\frac{1}{4}\|u\|^{4}-C\|u\|^{q},
\end{aligned}
$$

where $r>1$ is sufficiently close to $1,\|u\| \leq \sigma$, and $\kappa r \sigma^{2}<4 \pi^{2}$. So part (i) is proved if we choose $\|u\|=\rho>0$ small enough.

On the other hand, if $l \in\left(\mu_{1},+\infty\right)$, taking $\varepsilon>0$ such that $l-\varepsilon>\mu_{1}$ and using (31), we have

$$
\begin{aligned}
I\left(t \psi_{1}\right) \leq & \frac{1}{2} t^{2}\left\|\psi_{1}\right\|^{2}+\frac{1}{4}\left(1-\frac{l-\varepsilon}{\mu_{1}}\right) t^{4}\left\|\psi_{1}\right\|^{4} \\
& +B_{1}|\Omega| \longrightarrow-\infty \text { as } t \longrightarrow-\infty .
\end{aligned}
$$

Thus part (ii) is proved. By exactly slight modification to the proof above, we can prove (ii) if $l=+\infty$. By exactly slight modification to the proof above, we can prove (ii) if $l=+\infty$.

Lemma 12. For the functional I defined by (19), if $u_{n}(x) \leq 0$, a.e. $x \in \Omega, n \in \mathbb{N}$, and

$$
\left\langle I^{\prime}\left(u_{n}\right), u_{n}\right\rangle \longrightarrow 0 \quad \text { as } n \longrightarrow \infty
$$

then there exists a subsequence, still denoted by $\left\{u_{n}\right\}$, such that

$$
I\left(t u_{n}\right) \leq \frac{t^{2}}{2}\left\|u_{n}\right\|^{2}+\frac{1+t^{4}}{4 n}+I\left(u_{n}\right), \quad \forall t \geq 0, n \in \mathbb{N}
$$

Proof. Since $\left\langle I^{\prime}\left(u_{n}\right), u_{n}\right\rangle \rightarrow 0$ as $n \rightarrow \infty$, for a suitable subsequence, we may assume that

$$
\begin{aligned}
-\frac{1}{n}< & \left\langle I^{\prime}\left(u_{n}\right), u_{n}\right\rangle=\left(1+\left\|u_{n}\right\|^{2}\right)\left\|u_{n}\right\|^{2} \\
& -\int_{\Omega} f\left(x, u_{n}(x)\right) u_{n} d x<\frac{1}{n}, \quad \forall n .
\end{aligned}
$$

We claim that, for any $t \geq 0$ and $n \in \mathbb{N}$,

$$
\begin{aligned}
I\left(t u_{n}\right) \leq & \frac{1}{2} t^{2}\left\|u_{n}\right\|^{2}+\frac{t^{4}}{4 n} \\
& +\int_{\Omega}\left\{\frac{1}{4} f\left(x, u_{n}(x)\right) u_{n}-F\left(x, u_{n}(x)\right)\right\} d x
\end{aligned}
$$

Indeed, for any $t \geq 0$, at fixed $x \in \Omega$ and $n \in \mathbb{N}$, if we set

$$
h(t)=\frac{1}{4} t^{4} f\left(x, u_{n}\right) u_{n}(x)-F\left(x, t u_{n}(x)\right),
$$

then

$$
\begin{array}{r}
t^{3} f\left(x, u_{n}\right) u_{n}(x)-f\left(x, t u_{n}\right) u_{n}(x) \\
=t^{3} u_{n}(x)\left\{f\left(x, u_{n}\right)-\frac{f\left(x, t u_{n}(x)\right)}{t^{3}}\right\} \\
h^{\prime}(t)=\left\{\begin{array}{ll}
\geq 0 & \text { for } 0<t \leq 1 \\
\leq 0 & \text { for } t \geq 1
\end{array} \text { by }\left(H_{4}\right) .\right.
\end{array}
$$

Hence

$$
h(t) \leq h(1), \quad \forall t \geq 0 .
$$


Therefore,

$$
\begin{aligned}
I\left(t u_{n}\right)= & \frac{1}{2} t^{2}\left\|u_{n}\right\|^{2}+\frac{1}{4} t^{4}\left\|u_{n}\right\|^{4}-\int_{\Omega} F\left(x, t u_{n}(x)\right) d x \\
< & \frac{1}{2} t^{2}\left\|u_{n}\right\|^{2}+\frac{1}{4} t^{4}\left\{\frac{1}{n}+\int_{\Omega} f\left(x, u_{n}(x)\right) u_{n}(x) d x\right\} \\
& -\int_{\Omega} F\left(x, t u_{n}(x)\right) d x \\
\leq & \frac{1}{2} t^{2}\left\|u_{n}\right\|^{2}+\frac{t^{4}}{4 n} \\
& +\int_{\Omega}\left\{\frac{1}{4} t^{4} f\left(x, u_{n}(x)\right) u_{n}(x)-F\left(x, t u_{n}(x)\right)\right\} d x \\
\leq & \frac{1}{2} t^{2}\left\|u_{n}\right\|^{2}+\frac{t^{4}}{4 n} \\
& +\int_{\Omega}\left\{\frac{1}{4} f\left(x, u_{n}(x)\right) u_{n}(x)-F\left(x, u_{n}(x)\right)\right\} d x
\end{aligned}
$$

and our claim (37) is proved.

On the other hand,

$$
\begin{aligned}
I\left(u_{n}\right)= & \frac{1}{2}\left\|u_{n}\right\|^{2}+\frac{1}{4}\left\|u_{n}\right\|^{4}-\int_{\Omega} F\left(x, u_{n}(x)\right) d x \\
\geq & \frac{1}{4}\left\{-\frac{1}{n}+\int_{\Omega} f\left(x, u_{n}(x)\right) u_{n}(x) d x\right\} \\
& -\int_{\Omega} F\left(x, u_{n}(x)\right) d x .
\end{aligned}
$$

That is,

$$
\int_{\Omega}\left\{\frac{1}{4} f\left(x, u_{n}(x)\right) u_{n}(x)-F\left(x, u_{n}(x)\right)\right\} d x \leq \frac{1}{4 n}+I\left(u_{n}\right) .
$$

Combining (37) and (43), we find that

$$
I\left(t u_{n}\right) \leq \frac{1}{2} t^{2}\left\|u_{n}\right\|^{2}+\frac{1+t^{4}}{4 n}+I\left(u_{n}\right), \quad \forall t \geq 0, n \in \mathbb{N} .
$$

\section{Proofs of the Main Results}

Proof of Theorem 3. By Lemma 9, the geometry conditions of Mountain Mass Theorem hold. So we only need to verify condition (PS). Let $\left\{u_{n}\right\} \subset E$ be a (PS) sequence such that, for every $n \in \mathbb{N}$,

$$
\begin{gathered}
\left|\frac{1}{2}\left\|u_{n}\right\|^{2}+\frac{1}{4}\|u\|^{4}-\int_{\Omega} F\left(x, u_{n}\right) d x\right| \leq c, \\
\left|\left(1+\left\|u_{n}\right\|^{2}\right) \int_{\Omega} \nabla u_{n} \nabla v d x-\int_{\Omega} f\left(x, u_{n}\right) v d x\right| \leq \varepsilon_{n}\|v\| \\
v \in E
\end{gathered}
$$

where $c>0$ is a positive constant and $\left\{\varepsilon_{n}\right\} \subset \mathbb{R}^{+}$is a sequence which converges to zero.

Step 1. In order to prove that $\left\{u_{n}\right\}$ has a convergence subsequence, we first show that it is a bounded sequence. To do this, we argue by contradiction assuming that, for a subsequence which we follow denoted by $\left\{u_{n}\right\}$, we have

$$
\left\|u_{n}\right\| \longrightarrow+\infty \quad \text { as } n \longrightarrow \infty \text {. }
$$

Without loss of generality, we can assume that $\left\|u_{n}\right\|>1$, for all $n \in \mathbb{N}$, and define $z_{n}=u_{n} /\left\|u_{n}\right\|$. Obviously, $\left\|z_{n}\right\|=1$, for all $n \in \mathbb{N}$, and then it is possible to extract a subsequence (denoted also by $\left\{z_{n}\right\}$ ) such that

$$
\begin{gathered}
z_{n} \rightarrow z_{0} \quad \text { in } E, \\
z_{n} \longrightarrow z_{0} \quad \text { in } L^{4}(\Omega), \\
z_{n}(x) \longrightarrow z_{0}(x) \quad \text { a.e. } x \in \Omega, \\
\left|z_{n}(x)\right| \leq q(x) \quad \text { a.e. } x \in \Omega,
\end{gathered}
$$

where $z_{0} \in E$ and $q \in L^{4}(\Omega)$. Dividing both sides of (46) by $\left\|u_{n}\right\|^{3}$, we obtain

$$
\begin{aligned}
& \left|\left(1+\left\|u_{n}\right\|^{2}\right)\left\|u_{n}\right\|^{-2} \int_{\Omega} \nabla z_{n} \nabla v d x-\int_{\Omega} \frac{f\left(x, u_{n}\right)}{\left\|u_{n}\right\|^{3}} v d x\right| \\
& \quad \leq \frac{\varepsilon_{n}}{\left\|u_{n}\right\|^{3}}\|v\|, \quad \forall v \in E .
\end{aligned}
$$

Passing to the limit we deduce from (48) that

$$
\lim _{n \rightarrow \infty} \int_{\Omega} \frac{f\left(x, u_{n}\right)}{\left\|u_{n}\right\|^{3}} v d x=\int_{\Omega} \nabla z_{0} \nabla v d x
$$

for all $v \in E$

Now we claim that $z_{0}(x) \leq 0$ for a.e. $x \in \Omega$. To verify this, let us observe that by choosing $v=z_{0}^{+}=\max \left\{z_{0}, 0\right\}$ in (53) we have

$$
\lim _{n \rightarrow \infty} \int_{\Omega^{+}} \frac{f\left(x, u_{n}\right)}{\left\|u_{n}\right\|^{3}} z_{0} d x=\int_{\Omega^{+}}\left|\nabla z_{0}\right|^{2} d x<+\infty
$$

where $\Omega^{+}=\left\{x \in \Omega \mid z_{0}(x)>0\right\}$. But, on the other hand, from $\left(\mathrm{H}_{2}\right)$ and $\left(\mathrm{H}_{3}\right)$,

$$
\frac{f\left(x, u_{n}(x)\right)}{\left\|u_{n}\right\|^{3}} z_{0}(x) \geq\left(-l q(x)^{3}-K_{1}\right) z_{0}(x), \quad \text { a.e. } x \in \Omega,
$$

for some positive constant $K_{1}>0$. Moreover, using $\lim _{n \rightarrow \infty} u_{n}(x)=+\infty$, for a.e. $x \in \Omega^{+},(50)$, and the superlinearity of $f$ (see $\left(H_{3}\right)$ ), we also deduce

$$
\begin{aligned}
\lim _{n \rightarrow \infty} \frac{f\left(x, u_{n}(x)\right)}{\left\|u_{n}\right\|^{3}} z_{0}(x) & =\lim _{n \rightarrow \infty} \frac{f\left(x, u_{n}(x)\right)}{u_{n}^{3}} z_{n}(x)^{3} z_{0}(x) \\
& =+\infty, \quad \text { a.e. } x \in \Omega^{+}
\end{aligned}
$$



that

Therefore, if $\left|\Omega^{+}\right|>0$, by the Fatou lemma, we will obtain

$$
\lim _{n \rightarrow \infty} \int_{\Omega^{+}} \frac{f\left(x, u_{n}(x)\right)}{\left\|u_{n}\right\|^{3}} z_{0}(x) d x=+\infty
$$

which contradicts (54). Thus $\left|\Omega^{+}\right|=0$ and the claim is proved.

Clearly, $z_{0}(x) \not \equiv 0$. By $\left(H_{2}\right)$, there exists $c>0$ such that $\left|f\left(x, u_{n}\right)\right| /\left|u_{n}\right|^{3} \leq c$ for a.e. $x \in \Omega$. By using the Lebesgue dominated convergence theorem in (53), we have

$$
\left\|z_{0}\right\|^{2} \int_{\Omega} \nabla z_{0} \nabla v d x-\int_{\Omega} l z_{0}^{3} v d x=0
$$

for all $v \in E$. This contradicts our assumption; that is, $l \neq \mu_{i}$, for all $i \in \mathbb{N}$.

Step 2. Now, we prove that $\left\{u_{n}\right\}$ has a convergence subsequence. In fact, we can suppose that

$$
\begin{gathered}
u_{n} \rightarrow u \quad \text { in } E, \\
u_{n} \longrightarrow u \quad \text { in } L^{q}(\Omega), \quad \forall 1 \leq q<6, \\
u_{n}(x) \longrightarrow u(x) \quad \text { a.e. } x \in \Omega .
\end{gathered}
$$

Now, since $f$ has the subcritical growth on $\Omega$, for every $\epsilon>0$, we can find a constant $C(\epsilon)>0$ such that

$$
f(x, s) \leq C(\epsilon)+\epsilon|s|^{5}, \quad \forall(x, s) \in \Omega \times \mathbb{R} .
$$

Then

$$
\begin{aligned}
& \left|\int_{\Omega} f\left(x, u_{n}\right)\left(u_{n}-u\right) d x\right| \\
& \leq C(\epsilon) \int_{\Omega}\left|u_{n}-u\right| d x+\epsilon \int_{\Omega}\left|u_{n}-u\right|\left|u_{n}\right|^{5} d x \\
& \leq C(\epsilon) \int_{\Omega}\left|u_{n}-u\right| d x \\
& \quad+\epsilon\left(\int_{\Omega}\left(\left|u_{n}\right|^{5}\right)^{6 / 5} d x\right)^{5 / 6}\left(\int_{\Omega}\left|u_{n}-u\right|^{6}\right)^{1 / 6} \\
& \quad \leq C(\epsilon) \int_{\Omega}\left|u_{n}-u\right| d x+\epsilon C(\Omega) .
\end{aligned}
$$

Similarly, since $u_{n} \rightarrow u$ in $E, \int_{\Omega}\left|u_{n}-u\right| d x \rightarrow 0$. Since $\epsilon>0$ is arbitrary, we can conclude that

$$
\int_{\Omega}\left(f\left(x, u_{n}\right)-f(x, u)\right)\left(u_{n}-u\right) d x \longrightarrow 0 \quad \text { as } n \longrightarrow \infty .
$$

By (46), we have

$$
\left\langle I^{\prime}\left(u_{n}\right)-I^{\prime}(u),\left(u_{n}-u\right)\right\rangle \longrightarrow 0 \text { as } n \longrightarrow \infty .
$$

From (62) and (63), we obtain

$$
\left\|u_{n}\right\| \longrightarrow\|u\| \quad \text { as } n \longrightarrow \infty \text {. }
$$

So we have $u_{n} \rightarrow u$ in $E$ which means that $I$ satisfies (PS).
Proof of Theorem 4. Since $l=\lambda_{1}$, obviously, Lemma 9 (i) holds. We only need to show that Lemma 9 (ii) holds. Let $u=t \psi_{1}$. Using the condition $\left(H_{3}\right)$, then there exists $M>0$ large enough such that

$$
F(x, t) \geq M t^{4}-c
$$

for all $x \in \Omega$ and $t$ large enough. So we have

$$
\begin{aligned}
I\left(t \psi_{1}\right) \leq & \frac{1}{2} t^{2}\left\|\psi_{1}\right\|^{2}+\frac{1}{4} t^{4}\left\|\psi_{1}\right\|^{4}-M t^{4}\left\|\psi_{1}\right\|^{4} \\
& +C \longrightarrow-\infty \text { as } t \longrightarrow+\infty .
\end{aligned}
$$

By Proposition 2, there exists a sequence $\left\{u_{n}\right\} \subset E$ such that

$$
I\left(u_{n}\right)=\frac{1}{2}\left\|u_{n}\right\|^{2}+\frac{1}{4}\left\|u_{n}\right\|^{4}-\int_{\Omega} F\left(x, u_{n}\right) d x=c+\circ(1),
$$

$$
\left(1+\left\|u_{n}\right\|\right)\left\|I^{\prime}\left(u_{n}\right)\right\|_{E^{*}} \longrightarrow 0 \quad \text { as } n \longrightarrow \infty .
$$

Clearly, (68) implies that

$$
\begin{aligned}
\left\langle I^{\prime}\left(u_{n}\right), u_{n}\right\rangle= & \left\|u_{n}\right\|^{4}+\left\|u_{n}\right\|^{2} \\
& -\int_{\Omega} f\left(x, u_{n}(x)\right) u_{n} d x=\circ(1) .
\end{aligned}
$$

To complete our proof, we first need to verify that $\left\{u_{n}\right\}$ is bounded in $E$. Similar to the proof of Theorem 3, we have $z_{0}(x) \leq 0, x \in \Omega, z_{0}(x) \not \equiv 0$, and

$$
\left\|z_{0}\right\|^{2} \int_{\Omega} \nabla z_{0} \nabla v d x-\int_{\Omega} l z_{0}^{3} v d x=0
$$

for all $v \in E$. By maximum principle, $z_{0}<0$ is an eigenfunction of $\mu_{1}$; then $\left|u_{n}(x)\right| \rightarrow \infty$ for a.e. $x \in \Omega$. By our assumptions, we have

$$
\lim _{n \rightarrow \infty}\left(f\left(x, u_{n}(x)\right) u_{n}(x)-4 F\left(x, u_{n}(x)\right)\right)=+\infty
$$

uniformly in $x \in \Omega$, which implies that

$$
\int_{\Omega}\left(f\left(x, u_{n}(x)\right) u_{n}(x)-4 F\left(x, u_{n}(x)\right)\right) d x \longrightarrow+\infty
$$

as $n \longrightarrow \infty$.

On the other hand, (69) implies that

$$
4 I\left(u_{n}\right)-\left\langle I^{\prime}\left(u_{n}\right), u_{n}\right\rangle \longrightarrow 4 c \text { as } n \longrightarrow \infty \text {. }
$$

Thus

$$
\int_{\Omega}\left(f\left(x, u_{n}\right) u_{n}-4 F\left(x, u_{n}\right)\right) d x \longrightarrow-\infty \quad \text { as } n \longrightarrow \infty,
$$

which contradicts (72). Hence $\left\{u_{n}\right\}$ is bounded. According to the Step 2 proof of Theorem 3, we have $u_{n} \rightarrow u$ in $E$ which means that $I$ satisfies $\left(C_{c}\right)$. 
Proof of Theorem 5. By Lemma 9 and Proposition 2, (67)(69) hold. We still can prove that $\left\{u_{n}\right\}$ is bounded in $E$. Assume that $\left\|u_{n}\right\| \rightarrow+\infty$ as $n \rightarrow \infty$. Similar to the proof of Theorem 3, we have $z_{0}(x) \leq 0$ and when $z_{0}(x)<0$, $u_{n}=z_{n}\left\|u_{n}\right\| \rightarrow-\infty$ as $n \rightarrow \infty$. Let

$$
s_{n}=\frac{m}{\left\|u_{n}\right\|}, \quad w_{n}=s_{n} u_{n}=\frac{m u_{n}}{\left\|u_{n}\right\|},
$$

where $m=\sqrt{2}\left[c^{1 / 2}+c^{1 / 4}\right]$. Since $\left\{w_{n}\right\}$ is bounded in $E$, it is possible to extract a subsequence (denoted also by $\left\{w_{n}\right\}$ ) such that

$$
\begin{gathered}
w_{n} \rightarrow w_{0} \quad \text { in } E, \\
w_{n} \longrightarrow w_{0} \quad \text { in } \quad L^{4}(\Omega), \\
w_{n}(x) \longrightarrow w_{0}(x) \quad \text { a.e. } x \in \Omega, \\
\left|w_{n}(x)\right| \leq h(x) \quad \text { a.e. } x \in \Omega,
\end{gathered}
$$

where $w_{0} \in E$ and $h \in L^{4}(\Omega)$.

If $\left\|u_{n}\right\| \rightarrow+\infty$ as $n \rightarrow \infty$, then $w_{0}(x) \equiv 0$. In fact, letting $\Omega^{-}=\left\{x \in \Omega: w_{0}(x)<0\right\}$ and noticing $l=+\infty$, it follows from $\left(\mathrm{H}_{3}\right)$ that

$$
\frac{f\left(x, u_{n}\right)}{\left|u_{n}\right|^{2} u_{n}} \geq M \text { uniformly for all } x \in \Omega^{-},
$$

where $M$ is a large enough constant. Therefore, by (69) and (75), we have

$$
\begin{aligned}
m^{4} & =\lim _{n \rightarrow \infty}\left\|w_{n}\right\|^{4} \\
& =\lim _{n \rightarrow \infty} \int_{\Omega} \frac{f\left(x, u_{n}\right)}{\left|u_{n}\right|^{2} u_{n}}\left|w_{n}\right|^{4} d x \\
& \geq \lim _{n \rightarrow \infty} \int_{\Omega^{-}} \frac{f\left(x, u_{n}\right)}{\left|u_{n}\right|^{2} u_{n}}\left|w_{n}\right|^{4} d x \\
& \geq \operatorname{Mim}_{n \rightarrow \infty} \int_{\Omega^{-}}\left|w_{0}\right|^{4} d x .
\end{aligned}
$$

So $w_{0} \equiv 0$ for a.e. $x \in \Omega$. But if $w_{0} \equiv 0$, then $\int_{\Omega} F\left(x, w_{n}\right) d x \rightarrow$ 0 . Hence

$$
I\left(w_{n}\right)=\frac{m^{2}}{2}+\frac{m^{4}}{4} .
$$

On the other hand, by $\left\|u_{n}\right\| \rightarrow \infty$ as $n \rightarrow \infty$, we have $s_{n} \rightarrow$ 0 as $n \rightarrow \infty$. From Lemma 12 and (67), we get

$$
\begin{aligned}
I\left(w_{n}\right) & =I\left(s_{n} u_{n}\right) \\
& \leq \frac{m^{2}}{2}+\frac{1+\left(s_{n}\right)^{4}}{4 n}+I\left(u_{n}\right) \\
& \leq c+\frac{m^{2}}{2}, \text { as } n \longrightarrow \infty .
\end{aligned}
$$

Obviously, it contradicts (79). So $\left\{u_{n}\right\}$ is bounded in $E$. According to the Step 2 proof of Theorem 3, we have $u_{n} \rightarrow u$ in $E$ which means that $I$ satisfies $\left(C_{c}\right)$.
Proof of Theorem 6. By Lemma 11, the geometry conditions of Mountain Pass Theorem hold. So we only need to verify condition (PS). Similar to the Step 1 proof of Theorem 3, we easily know that (PS) sequence $\left\{u_{n}\right\}$ is bounded in $E$. Next, we prove that $\left\{u_{n}\right\}$ has a convergence subsequence. Without loss of generality, suppose that

$$
\begin{gathered}
\left\|u_{n}\right\| \leq \beta, \\
u_{n} \rightarrow u \quad \text { in } E, \\
u_{n} \longrightarrow u \quad \text { in } L^{q}(\Omega), \quad \forall q \geq 1, \\
u_{n}(x) \longrightarrow u(x) \quad \text { a.e. } x \in \Omega .
\end{gathered}
$$

Now, since $f$ has the subcritical exponential growth (SCE) on $\Omega$, we can find a constant $C_{\beta}>0$ such that

$$
|f(x, t)| \leq C_{\beta} \exp \left(\frac{\alpha_{2}}{2 \beta^{2}}|t|^{2}\right), \quad \forall(x, t) \in \Omega \times \mathbb{R} .
$$

Thus, by the Moser-Trudinger inequality (see Lemma 10),

$$
\begin{aligned}
& \left|\int_{\Omega} f\left(x, u_{n}\right)\left(u_{n}-u\right) d x\right| \\
& \quad \leq C\left(\int_{\Omega} \exp \left(\frac{\alpha_{2}}{\beta^{2}}\left|u_{n}\right|^{2}\right) d x\right)^{1 / 2}\left|u_{n}-u\right|_{2} \\
& \quad \leq C\left(\int_{\Omega} \exp \left(\frac{\alpha_{2}}{\beta^{2}}\left\|u_{n}\right\|^{2}\left|\frac{u_{n}}{\left\|u_{n}\right\|}\right|^{2}\right) d x\right)^{1 / 2}\left|u_{n}-u\right|_{2} \\
& \quad \leq C\left|u_{n}-u\right|_{2} \rightarrow 0 .
\end{aligned}
$$

Similar to the last proof of Theorem 3, we have $u_{n} \rightarrow u$ in $E$ which means that $I$ satisfies (PS).

Proof of Theorem 7. Combining the proof of Theorems 4 and 6 , we easily prove it.

Proof of Theorem 8. Combining the proof of Theorems 5 and 6 , we easily prove it.

\section{Conflict of Interests}

The authors declare that there is no conflict of interests regarding the publication of this paper.

\section{Acknowledgments}

The authors would like to thank the referees for valuable comments and suggestions in improving this paper. This work was supported by the National Natural Science Foundation of China (Grant no. 11101319) and the Planned Projects for Postdoctoral Research Funds of Jiangsu Province (Grant no. 1301038C).

\section{References}

[1] C. O. Alves, F. J. S. A. Corrêa, and T. F. Ma, "Positive solutions for a quasilinear elliptic equation of Kirchhoff type," Computers \& Mathematics with Applications, vol. 49, no. 1, pp. 85-93, 2005. 
[2] G. Kirchhoff, Vorlesungen Über Mechanik, Teubner, Leipzig, Germany, 1883.

[3] A. Arosio and S. Panizzi, "On the well-posedness of the Kirchhoff string," Transactions of the American Mathematical Society, vol. 348, no. 1, pp. 305-330, 1996.

[4] M. Chipot and B. Lovat, "Some remarks on nonlocal elliptic and parabolic problems," Nonlinear Analysis: Theory, Methods \& Applications, vol. 30, no. 7, pp. 4619-4627, 1997.

[5] P. D’Ancona and S. Spagnolo, "Global solvability for the degenerate Kirchhoff equation with real analytic data," Inventiones Mathematicae, vol. 108, no. 2, pp. 247-262, 1992.

[6] T. F. Ma and J. E. M. Rivera, "Positive solutions for a nonlinear nonlocal elliptic transmission problem," Applied Mathematics Letters, vol. 16, no. 2, pp. 243-248, 2003.

[7] X. He and W. Zou, "Infinitely many positive solutions for Kirchhoff-type problems," Nonlinear Analysis: Theory, Methods \& Applications, vol. 70, no. 3, pp. 1407-1414, 2009.

[8] K. Perera and Z. Zhang, "Nontrivial solutions of Kirchhoff-type problems via the Yang index," Journal of Differential Equations, vol. 221, no. 1, pp. 246-255, 2006.

[9] Z. Zhang and K. Perera, "Sign changing solutions of Kirchhoff type problems via invariant sets of descent flow," Journal of Mathematical Analysis and Applications, vol. 317, no. 2, pp. 456463, 2006.

[10] A. Mao and Z. Zhang, "Sign-changing and multiple solutions of Kirchhoff type problems without the P.S. condition," Nonlinear Analysis: Theory, Methods \& Applications, vol. 70, no. 3, pp. 1275-1287, 2009.

[11] D. Arcoya and S. Villegas, "Nontrivial solutions for a Neumann problem with a nonlinear term asymptotically linear at $-\infty$ and superlinear at $+\infty$," Mathematische Zeitschrift, vol. 219, no. 4, pp. 499-513, 1995.

[12] D. G. de Figueiredo and B. Ruf, "On a superlinear SturmLiouville equation and a related bouncing problem," Journal für die Reine und Angewandte Mathematik, vol. 421, pp. 1-22, 1991.

[13] K. Perera, "Existence and multiplicity results for a SturmLiouville equation asymptotically linear at $-\infty$ and superlinear at $+\infty$," Nonlinear Analysis: Theory, Methods \& Applications, vol. 39, no. 6, pp. 669-684, 2000.

[14] D. Motreanu, V. V. Motreanu, and N. S. Papageorgiou, "Multiple solutions for Dirichlet problems which are superlinear at $+\infty$ and (sub-)linear at $-\infty$," Communications in Applied Analysis, vol. 13, no. 3, pp. 341-357, 2009.

[15] B. Cheng and X. Wu, "Existence results of positive solutions of Kirchhoff type problems," Nonlinear Analysis: Theory, Methods \& Applications, vol. 71, no. 10, pp. 4883-4892, 2009.

[16] Z. Liu and Z.-Q. Wang, "On the Ambrosetti-Rabinowitz superlinear condition," Advanced Nonlinear Studies, vol. 4, no. 4, pp. 563-574, 2004.

[17] A. Ambrosetti and P. H. Rabinowitz, "Dual variational methods in critical point theory and applications," vol. 14, pp. 349-381, 1973.

[18] D. G. Costa and O. H. Miyagaki, "Nontrivial solutions for perturbations of the $p$-Laplacian on unbounded domains," Journal of Mathematical Analysis and Applications, vol. 193, no. 3, pp. 737-755, 1995.

[19] N. S. Trudinger, "On imbeddings into Orlicz spaces and some applications," Journal of Mathematics and Mechanics, vol. 17, pp. 473-483, 1967.

[20] J. Moser, "A sharp form of an inequality by N. Trudinger," Indiana University Mathematics Journal, vol. 20, pp. 1077-1092, 1971. 


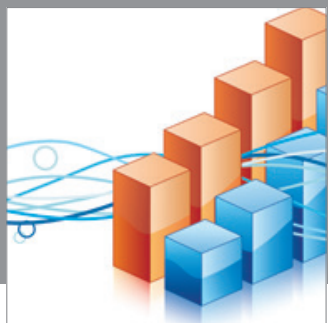

Advances in

Operations Research

mansans

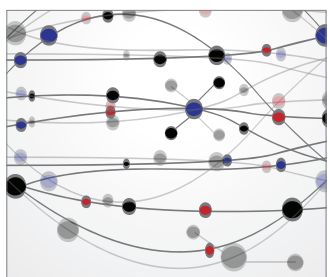

The Scientific World Journal
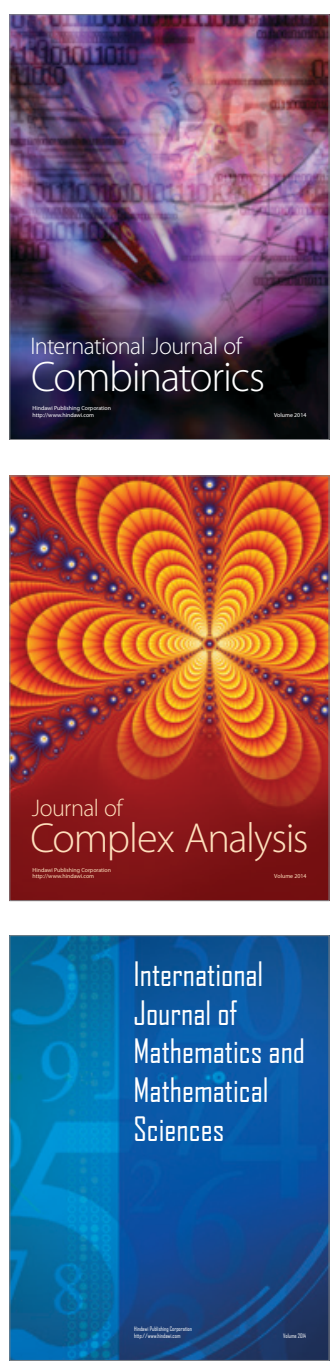
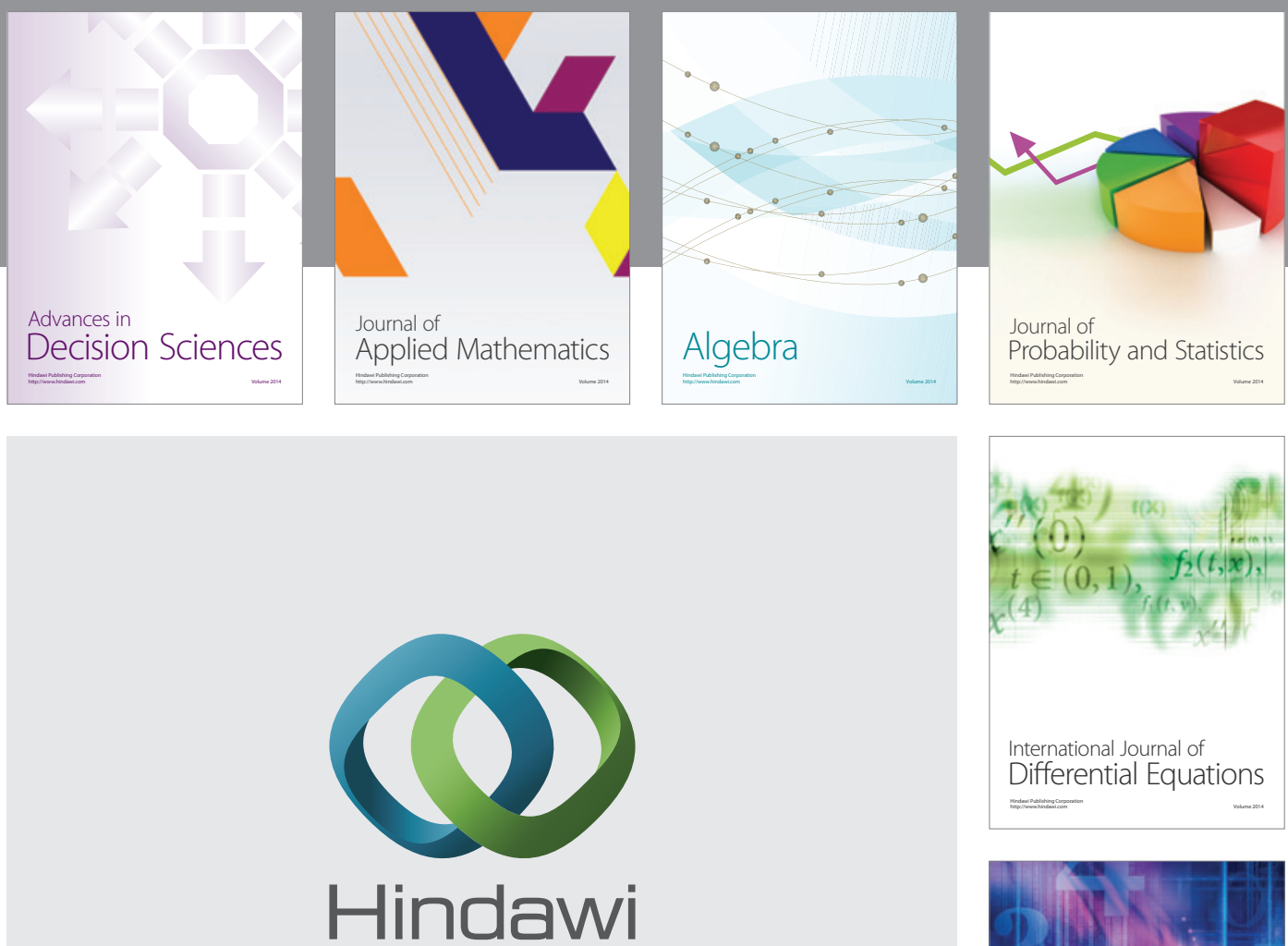

Submit your manuscripts at http://www.hindawi.com
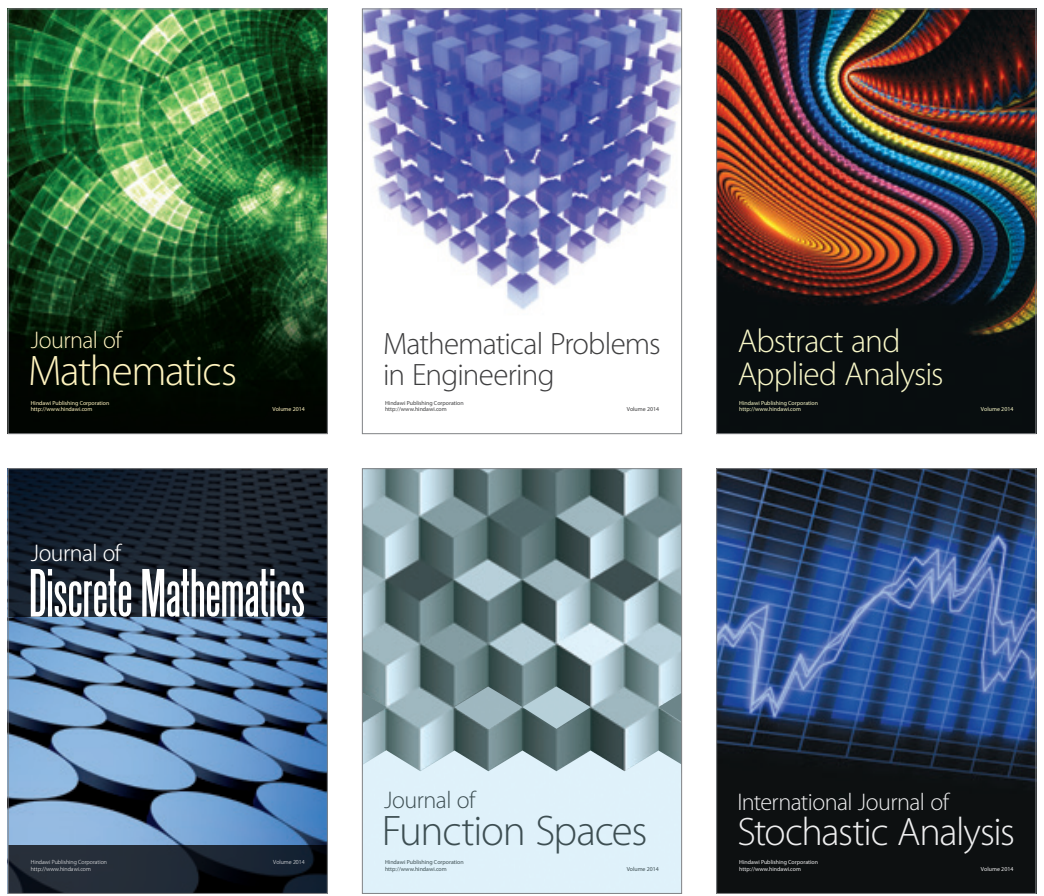

Journal of

Function Spaces

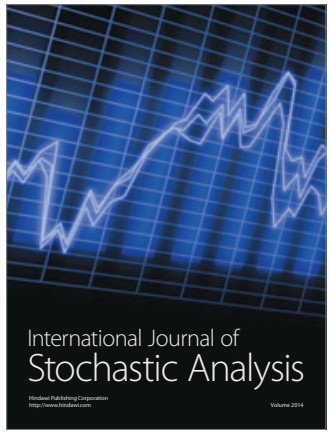

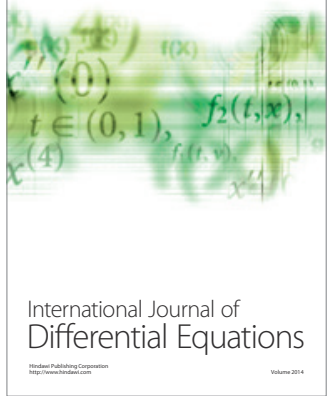
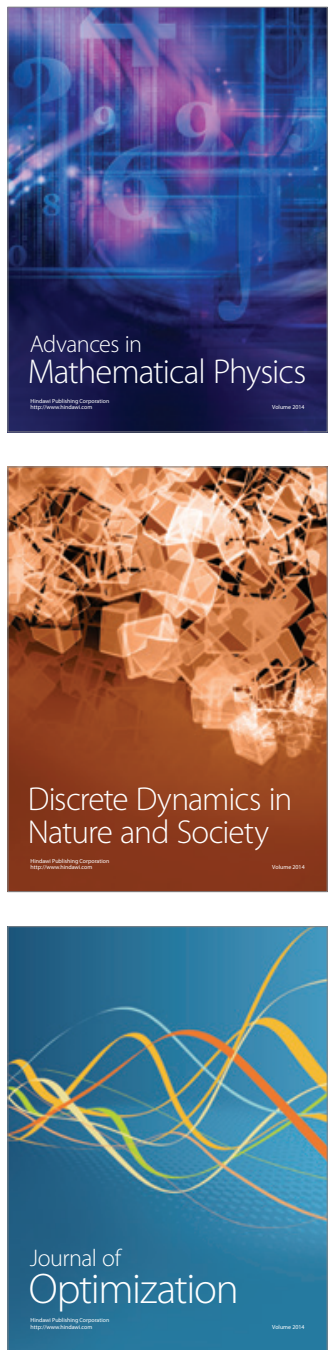